\title{
Cholera toxin promotes the generation of semi-mature porcine monocyte-derived dendritic cells that are unable to stimulate $\mathbf{T}$ cells
}

\author{
Diane BIMczoK ${ }^{\mathrm{a} *}$, Henriette RAU ${ }^{\mathrm{a}}$, Nicole WUNDRACK ${ }^{\mathrm{b}}$, Michael \\ NAUmAnN $^{\mathrm{b}}$, Hermann-Josef RothKöTteR ${ }^{\mathrm{a}}$, Kenneth McCullough ${ }^{\mathrm{c}}$, \\ Artur SUMMERFIELD ${ }^{\mathrm{c}}$ \\ ${ }^{a}$ Institute of Anatomy, Otto-von-Guericke University Magdeburg, Leipziger Strasse 44, \\ 39120 Magdeburg, Germany \\ ${ }^{\mathrm{b}}$ Institute of Experimental Internal Mediane, Otto-von-Guiricke University, Magdeburg, Germany \\ ${ }^{\mathrm{c}}$ Institute of Virology and Immunoprophylaxis (IVI), Mittelhäusenn, Switzerland
}

(Received 11 October 2006; accepted 16 February 2007)

\begin{abstract}
Cholera toxin (Ctx) is a powerful mucosal adjuvant with potential applications for oral vaccination of swine. Dendritic cells (DC) play a key role in the decision between immunity and tolerance, and are likely target cells for mediating Ctx functions in vivo. Therefore, we examined the capacity of Ctx to enhance stimulatory activity of porcine monocyte-derived DC (MoDC). Ctx promoted the development of a semi-mature DC phenotype, with decreased levels of MHC class II and CD40, but increased CD80/86 expression. These changes were associated with activation of extracellular signal-regulated kinase (ERK), but not NFkB or c-Jun N-terminal kinase (JNK). Functionally, Ctx-priming greatly diminished T cell stimulatory capacity both in antigen-specific and superantigen-induced proliferation assays. The lower proliferation rate was not due to increased apoptosis of either DC or T cells. Ctx suppressed TNF $\alpha$ secretion by MoDC, but induced IL-10 production. The observed effects on T cell proliferation could only be partially mimicked by IL-10 alone. However, addition of recombinant TNF $\alpha$ to co-cultures of Ctx-primed MoDC and lymphocytes restored lymphocyte proliferation in a concentration-dependent manner. Ctx-primed DC were not actively tolerogenic, since they could not suppress proliferative $\mathrm{T}$ cell reactions induced by untreated DC.
\end{abstract}

pig / dendritic cell / cholera toxin / adjuvant

\section{INTRODUCTION}

Oral vaccine delivery is a convenient mode of immunisation because of easy administration and good acceptability. It can also be used for bait vaccination

\footnotetext{
* Corresponding author: dianebimczok@yahoo.de
}

of wildlife, e.g. immunisation of wild boar against classical swine fever [24,33]. To prevent unnecessary immune reactions against harmless food proteins, the default reaction to orally applied, nonreplicating antigens is the development of tolerance [45]. This has to be overriden by co-application of mucosal adjuvants. 
Cholera toxin (Ctx) is a potent mucosal adjuvant that has been used successfully in a large number of murine studies $[10,13,14,29]$. Unfortunately, the high toxicity of native Ctx in humans precludes the clinical use of Ctx [32]. In the pig, studies investigating the potential of Ctx as a mucosal adjuvant yielded contradictory results: co-administration of $\mathrm{Ctx}$ together with keyhole limpet hemocyanin (KLH) did not evoke any detectable antibody response to KLH [16]. In contrast, Verdonck et al. [48] could demonstrate that co-application of Ctx with Escherichia coli F4 fimbriae improved both humoral and cellular response to $\mathrm{F} 4$, and reduced $E$. coli excretion in a challenge experiment.

The results from studies in humans and mice indicate that the adjuvanticity of $\mathrm{Ctx}$ may largely depend on its interaction with dendritic cells (DC), which mature in the presence of Ctx [18, 19, 22]. DC are professional antigen-presenting cells (APC) with the unique ability to activate naïve T cells [4]. Specific DC subsets and maturation stages of DC can direct the immune system towards either tolerance or immunity $[7,15]$. In the pig, the effects of $\mathrm{Ctx}$ on alveolar macrophages have been analysed [17]. In vitro stimulation with Ctx caused an increased expression of inflammatory cytokines and co-stimulatory molecules, indicating macrophage activation, which could promote an active immune response against other antigens. However, the functional roles of alveolar macrophages as APC clearly differ from those of DC. Furthermore, Ctx can also confer immunomodulatory effects through DC [29].

To further analyse the adjuvant mechanism of Ctx in pigs, we focussed on DC as key antigen presenting cells. Although DC located in the intestinal lamina propria, Peyer's Patches and mesenteric lymph nodes are the most likely target cells for mucosal adjuvants, these cells are not easily accessible for in vitro studies. In con- trast, DC generated from blood monocytes (monocyte-derived dendritic cells, MoDC) are a well established and stable model system in swine research $[8,23,35,37,38]$. Therefore porcine MoDC were used for an exploratory in vitro study to investigate the effects of Ctx on porcine DC. Our data show that, in contrast to previous observations with human MoDC, Ctx induces a unique semi-mature MoDC phenotype with impaired stimulatory capacity in the porcine system.

\section{MATERIALS AND METHODS}

\subsection{Animals and blood samples}

Blood samples were taken from specific pathogen-free Landrace pigs kept at the Institute of Virology and Immunoprophylaxis (IVI), Switzerland, using citrate as an anticoagulant. Alternatively, heparinised blood was obtained from Landrace pigs kept under standard conditions at the Institute of Medical Technology Magdeburg GmbH, Germany. To avoid stress reactions, pigs were sedated with Ketamine/Xylacine (standard dose) during sampling procedures in some instances. All animal experiments were in accordance with local animal welfare regulations, permission numbers 19/05 (Switzerland) and 203.6.1-42502/2-641 (Germany).

\subsection{Isolation and culture of cells}

PBMC were isolated from whole blood by Ficoll density gradient centrifugation (Pharmacia, Uppsala, Sweden). Monocytes were further enriched to $>95 \%$ purity by positive magnetic bead selection (MACS, Miltenyi-Biotec, Bergisch Gladbach, Germany) using an antibody directed against CD172a (mAb 74-22-15A) and goat anti-mouse IgG microbeads together with LS separation columns (MACS). Cells were then cultured at $39{ }^{\circ} \mathrm{C}, 5 \%$ 
$\mathrm{CO}_{2}$ with phenol-red free Dulbecco's modified Eagle's Medium (DMEM; Invitrogen, Basel, Switzerland) supplemented with porcine serum $(10 \% \mathrm{v} / \mathrm{v}$; Sigma, Buchs, Switzerland), recombinant porcine (rp) GM-CSF (150 ng/mL, kindly provided by $\mathrm{Dr} \mathrm{S}$. Inumaru, Institute for Animal Health, Ibaraki, Japan, or purchased from Biosource, Camarillo, CA, USA) and rp IL-4 $(50 \mathrm{ng} / \mathrm{mL}$; prepared at the IVI, or from Biosource) to generate $\mathrm{MoDC}$ as described previously $[8,49]$. Cultures were supplemented with fresh cytokines after three days and further processed as indicated.

\subsection{Stimulation of MoDC cultures}

Ctx or Ctx-B-subunit (Sigma, Taufkirchen, Germany) were added to MoDC cultures for 24 to $48 \mathrm{~h}$ on day 3 at final concentrations of $1 \mu \mathrm{g} / \mathrm{mL}$, following previous studies with human and murine DC $[2,18,19,22]$. To exclude the influence of LPS contamination of the toxin preparation, Ctx detoxified with Detoxi-Gel ${ }^{\mathrm{TM}}$ AffinityPak ${ }^{\mathrm{TM}}$ Columns (Perbio Science, Bonn, Germany) was used in some experiments. Negative controls were prepared by pre-incubation of $\mathrm{Ctx}$ with its soluble receptor, GM1 (10 $\mu \mathrm{g} / \mathrm{mL}$; Sigma). DC maturation was induced with different combinations of the following reagents: rp IFN $\alpha(1000 \mathrm{U} / \mathrm{mL}), \mathrm{rp}$ TNF $\alpha(10 \mathrm{ng} / \mathrm{mL})$, or LPS $(1 \mu \mathrm{g} / \mathrm{mL}$, Sigma). To analyse the influence of IL-10 on MoDC, cultures were stimulated with $1-1000 \mathrm{ng} / \mathrm{mL}$ of rp IL-10 (Biosource). IFN $\alpha$ was prepared at the IVI laboratory as described previously [3], and TNF $\alpha$ was kindly provided by Dr G. Bertoni, Bern [50] or purchased from NatuTec (Frankfurt, Germany).

\subsection{Monoclonal antibodies (mAbs) and phenotyping}

For phenotyping, mAbs against the following cell surface molecules were used: CD172a (mAb 74-22-15A; recognises SIRP $\alpha$, SWC3a), MHC class II (mAb MSA3), CD40 (mAb G28-5, anti-human), CCR1 (mAb 145, anti human, R\&D Systems, Abingdon, UK). A human CTLA4 muIg fusion protein (Ancell, Bayport, MN, USA) was used to detect expression of CD80 and CD86. Hybridomas for the mAbs 74-11-10, MSA3, and 74-22$15 \mathrm{~A}$ were donated by Prof. A. Saalmüller (University of Veterinary Medicine Vienna, Austria). Hybridoma supernatant for the mAb G28-5 was provided by T. Bedke (Medical School of Hanover, Germany).

For immunofluorescence labelling, the cells received the mAbs at pre-titrated saturating concentrations for $30 \mathrm{~min}$ at $4{ }^{\circ} \mathrm{C}$, then the appropriate isotype-specific conjugates (goat anti-mouse antibodies, coupled to FITC or PE; Southern Biotechnology, Birmingham, AL, USA) for another $15 \mathrm{~min}$ at $4{ }^{\circ} \mathrm{C}$. The cells were analysed with a FACSCalibur flow cytometer using CellQuest Pro ${ }^{\circledR}$ software (Becton Dickinson).

\subsection{Antigen uptake}

Uptake of soluble antigen was studied using $\mathrm{DQ}^{\mathrm{TM}}$ ovalbumin (OvaDQ, Molecular Probes, Leiden, Netherlands). MoDC were harvested from the plates and incubated for $30 \mathrm{~min}$ at $37^{\circ} \mathrm{C}$ with $10 \mu \mathrm{g} / \mathrm{mL}$ of OvaDQ in culture medium. The cells were then analysed by flow cytometry.

\subsection{MoDC proliferation assays}

Superantigen-dependent stimulation was analysed in a staphylococcal enterotoxin B (SEB) presentation assay. Autologous lymphocytes were enriched by depletion of CD172a positive cells from PBMC using the MACS system. MoDC pre-treated with Ctx and/or other stimulants were harvested, washed, and 
then incubated $1 \mathrm{~h}$ at $39{ }^{\circ} \mathrm{C}$ with SEB $(1 \mu \mathrm{g} / \mathrm{mL}$, Toxin Technology, Sarasota, FL, USA), followed by three washing steps. MoDC were then co-cultured at a ratio of 1:10 with 200000 lymphocytes per well in triplicates. After three days of culture, the cells were pulsed with $1 \mu \mathrm{Ci} /$ well $\left[{ }^{3} \mathrm{H}\right]$ methyl-thymidine (Moravek Biochemicals) for another $18 \mathrm{~h}$. Plates were harvested onto filter mats and counted in a 1450 MicroBeta $^{\circledR}$ Trilux counter (Wallac). Alternatively, cell proliferation was evaluated by BrdU-ELISA (Roche, Mannheim, Germany) according to the manufacturer's instructions, or by MTT-assay as described previously [11]. Proliferation index was calculated by dividing measured absorption of samples by absorption value of untreated lymphocytes.

Antigen-specific stimulation was assessed using lymphocytes from pigs primed with classical swine fever virus (CSFV) by infection with $10^{3}$ TCID $_{50}$ of CSFV strain Alfort [41]. MoDC cultures were pulsed with the following antigens for $26 \mathrm{~h}$, with $\mathrm{Ctx}$ added after $2 \mathrm{~h}$ if required: (1) CSFV strain Alfort (0.01 TCID $_{50} /$ cell), (2) mock antigen prepared from uninfected SK6 cells used to propagate the virus [27], (3) E2-protein of CSFV, baculovirus-expressed $(4 \mu \mathrm{g} / \mathrm{mL})$ produced as described elsewhere [36], or (4) CSFV-non-structural protein 3 (NS3)-protein of CSFV, E. coli-expressed ( $2 \mu \mathrm{g} / \mathrm{mL}$; kindly provided by Dr Hilde Revets, Free University Brussels, Belgium). MoDC were then co-cultured with autologous lymphocytes in the presence of the respective antigens for 3-4 days, and analysis of $\left[{ }^{3} \mathrm{H}\right]$ methyl-thymidine incorporation was performed as described above.

To examine the role of TNF $\alpha$ in MoDC-lymphocyte co-cultures, pretreated MoDC were loaded with SEB as described above and cultured with autologous lymphocytes in the presence of $\mathrm{rp} \mathrm{TNF} \alpha(0.1-10 \mathrm{ng})$. Proliferation was determined by BrdU-ELISA. For these experiments, $\beta$-mercaptoethanol ( $50 \mu \mathrm{mol}$, PAN Biotech, Aidenbach, Germany) was added to the medium, which resulted in considerably higher proliferation indices.

\subsection{Apoptosis assay}

Induction of apoptosis and cell death by Ctx was assessed using Annexin VFITC and propidiumiodide (Serotec, Oxford, UK). MoDC were stimulated with $1 \mu \mathrm{g}$ of Ctx for 2, 6 or $24 \mathrm{~h}$. Alternatively, co-cultures of pre-stimulated MoDC and lymphocytes were set up as described previously for the proliferation assays, and lymphocytes were analysed for apoptosis after 2, 6 or $24 \mathrm{~h}$ of co-culture. In positive control samples, apoptosis was induced by adding staurosporin (1 mM, Sigma). Samples were then stained with Annexin V-FITC following the manufacturer's protocol. After addition of propidiumiodide ( $5 \mu \mathrm{g} / \mathrm{mL})$, the cells were analysed with a FACSCalibur flow cytometer using CellQuest Pro ${ }^{\circledR}$ software (Becton Dickinson).

\subsection{Detection of cytokine production}

Cell-free culture supernatants of MoDC were collected for analysis of cytokines. To avoid a direct influence of the added stimulants on the PK15-15 cells in the TNF $\alpha$ bioassay, medium was exchanged twice after the first hour of incubation with the stimulants, and supernatants were collected after a total of 6,24 or 48 h. IL-10 and IL-6 were quantified using commercial ELISA kits (IL-10: Biosource; IL-6: R\&D Systems, Minneapolis, USA). Similarly, IL-12 levels were detected by ELISA as previously described [20]. TNF $\alpha$ production was analysed by bioassay as previously described by Bertoni et al. [6]. Briefly, PK15-15 cells (kindly provided by Dr G. Bertoni, Bern, Switzerland) grown 
in DMEM (Invitrogen, Basel, Switzerland) supplemented with $7.5 \%$ FCS (Sigma) were seeded at a density of $2 \times 10^{5}$ in 96 well plates. After over night culture at $37{ }^{\circ} \mathrm{C}, 5 \% \mathrm{CO}_{2}$, the cells were sensitised to $\mathrm{TNF} \alpha$-induced cell death with $3 \mu \mathrm{g} / \mathrm{mL}$ actinomycin D (Sigma, Taufkirchen, Germany) for $2 \mathrm{~h}$ before adding supernatant samples. The number of viable cells was analysed after $16 \mathrm{~h}$ using MTT, as described above. TNF $\alpha$ concentrations were calculated from a standard curve generated with rp TNF $\alpha$ (NatuTec, Frankfurt, Germany).

\subsection{SDS-PAGE and immunoblotting}

MoDC were stimulated for 10-90 min with $10 \mu \mathrm{g} / \mathrm{mL} \mathrm{Ctx}$ to ensure optimum loading of receptors during the short incubation time. The cells were then lysed, and proteins were subjected to SDSPAGE and immunoblotting as described previously [34]. Immunoblots were developed using enhanced chemiluminescence (ECL, Amersham Biosciences) according to the manufacturer's instructions. The following antibodies were used to detect the respective proteins: anti-I $\kappa \mathrm{B} \alpha$ (Santa Cruz Biotechnology, Santa Cruz, CA, USA), anti-phospho$\mathrm{I} \kappa \mathrm{B} \alpha(\mathrm{Ser} 32 / 36)$, anti-extracellular signalregulated kinase (ERK), anti-phosphoERK, and anti-phospho- c-Jun N-terminal kinase (JNK) (all Cell Signaling, Frankfurt, Germany).

\subsection{Analysis of the effect of secreted cytokines on lymphocyte proliferation}

To assess the direct effects of IL-10 on proliferation of porcine lymphocytes, 5$100 \mathrm{ng} / \mathrm{mL}$ of rp IL-10 (Biosource) were added to PBMC cultures $\left(1 \times 10^{5} /\right.$ well $)$ together with ConA (10 $\mu \mathrm{g} / \mathrm{mL}$; Sigma). Alternatively, to detect the influence of other factors secreted by MoDC, PBMC were incubated in the presence of cell-free culture supernatants collected 3-24 h after stimulation of the DC, and cell proliferation was measured by MTT-assay after 3 days of culture in DMEM supplemented with $10 \%$ of porcine serum at $39^{\circ} \mathrm{C}, 5 \% \mathrm{CO}_{2}$.

\subsection{Statistical analyses}

Statistical analyses were performed with Microsoft ${ }^{\circledR}$ Excel 2000 software. The results are given as mean \pm standard deviation. Differences between values were analysed for statistical significance with the Student's $t$-test and were considered significant at $P<0.05$.

\section{RESULTS}

\subsection{Influence of $\mathrm{Ctx}$ on expression of maturation markers by MoDC}

In order to evaluate DC maturation status, we analysed surface expression of MHC class II (MHC-II), CD40, CD80/CD86, and chemokine receptor CCR1 by flow cytometry. Stimulation of MoDC with LPS, TNF $\alpha$, LPS+TNF $\alpha$, or IFN $\alpha+\mathrm{TNF} \alpha$ ("maturation factors"), caused an increase in MHC-II, CD40 and CD80/86 expression, and a decrease in CCR1 expression. These changes are typically associated with functional DC maturation. Representative histograms are shown in Figure 1. Surprisingly, addition of cholera toxin (Ctx) to MoDC cultures decreased surface expression of both MHC-II and CD40. This effect was consistently observed, with a geometric mean fluorescence intensity reduction of MHC class II by $28 \% \pm 5 \%(P<0.001$, Student's $t$-test). The decreased expression of maturation markers became especially prominent in the presence of maturation factors, indicating inhibition of DC maturation by $\mathrm{Ctx}$. In clear contrast to the effect 

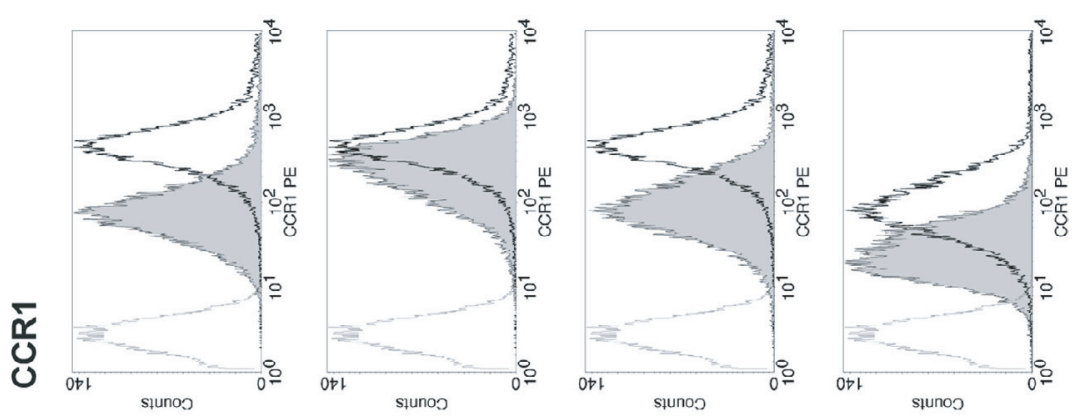

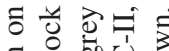
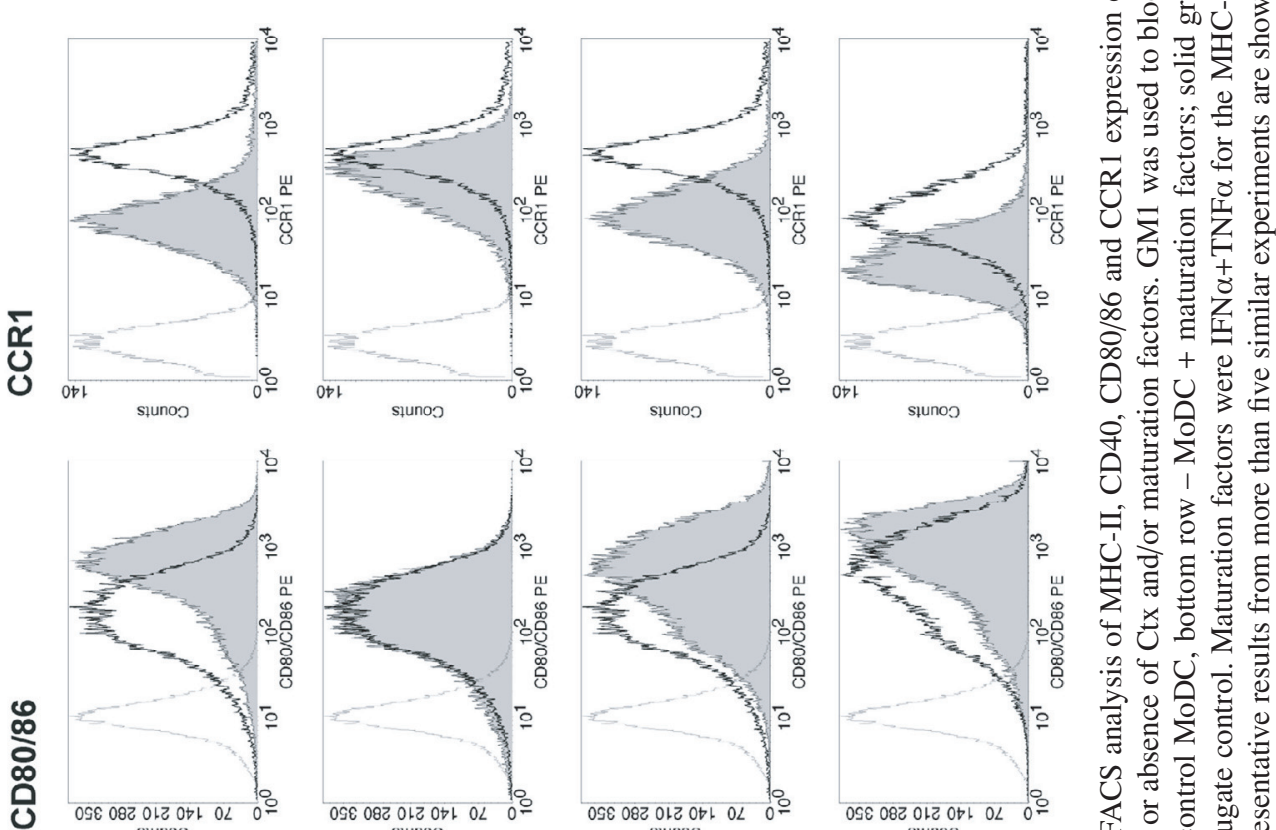

守苛完的

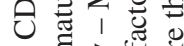

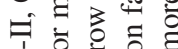

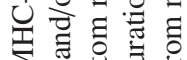
$\sum \widetilde{E} \vec{J}^{ \pm}$ प⿺辶寸

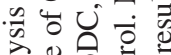

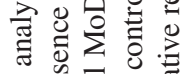

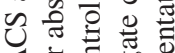
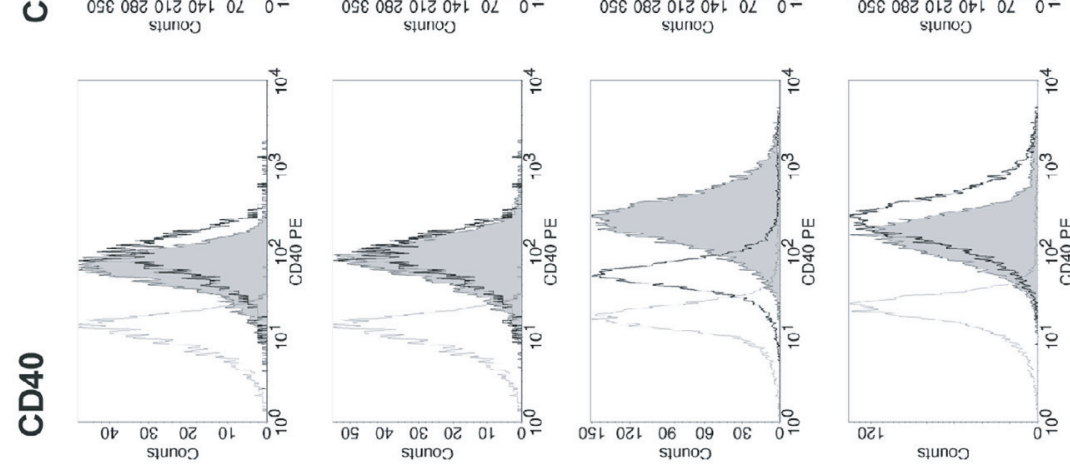

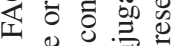
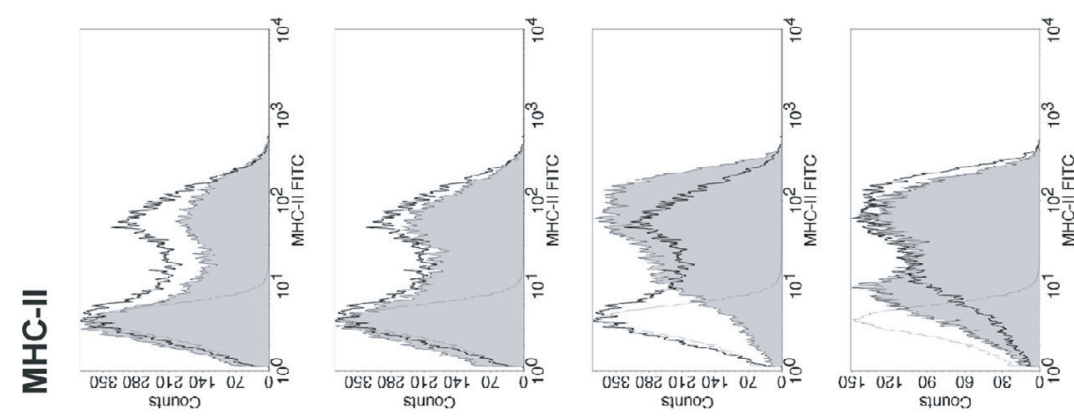

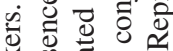

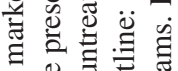
ฮิ

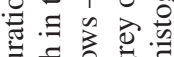

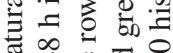
苂就

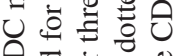

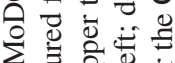

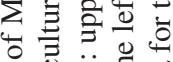

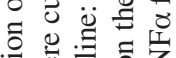

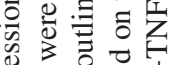
के ठ

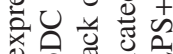
¿ ช $\unrhd . \Xi$ च कี

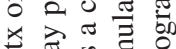

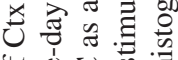
प⿺辶寸

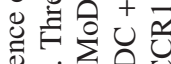
论。

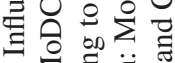

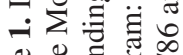
u!xol еләрочว เWอ + X75

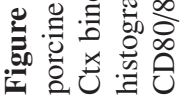


on MHC and CD40, Ctx caused an increased CD80/86 and decreased CCR 1 expression, consistent with maturation. In the presence of IFN $\alpha+\mathrm{TNF} \alpha$, Ctx had an additive effect on the modulation of CD80/86 and CCR1 expression. All effects were already seen after $24 \mathrm{~h}$, but became more prominent after $48 \mathrm{~h}$ of stimulation. In general, Ctx-induced effects on maturation marker expression could be largely abrogated if soluble GM1 receptor was added to the cultures, which demonstrates that the observed changes were Ctx-specific. Similarly, Ctx retained its activity after removal of LPS using Detoxi-Gel ${ }^{\mathrm{TM}}$ AffinityPak ${ }^{\mathrm{TM}}$ columns (data not shown), confirming that the observed effects were not caused by LPS-contamination of the Ctx preparation.

\subsection{Cholera toxin does not alter endocytic capacity of MoDC}

Antigen uptake is a typical feature of immature DC, and is therefore reduced after maturation. We tested the endocytic capacity of porcine MoDC after $48 \mathrm{~h}$ of culture in the presence of different stimulants, using the protein conjugate $\mathrm{DQ}^{\mathrm{TM}}$ Ovalbumin (OvaDQ) as marker substances. As shown in Figure 2, Ctx alone had no obvious effect on OvaDQ uptake. Likewise, although the maturation factors LPS $+\mathrm{TNF} \alpha$ caused the expected down-regulation of OvaDQ uptake, this was not further modified by the addition of Ctx to the cultures.

\subsection{Effects of Ctx on ERK and NF-кB activation}

We investigated the impact of Ctx on the inducible activation of ERK, JNK and NFkB in MoDC. As shown in Figure 3, phosphorylation of ERK was induced within 20 min and increased steadily within the indicated periods of time. In contrast to ERK activation, we could not

\section{Ova-DQ}
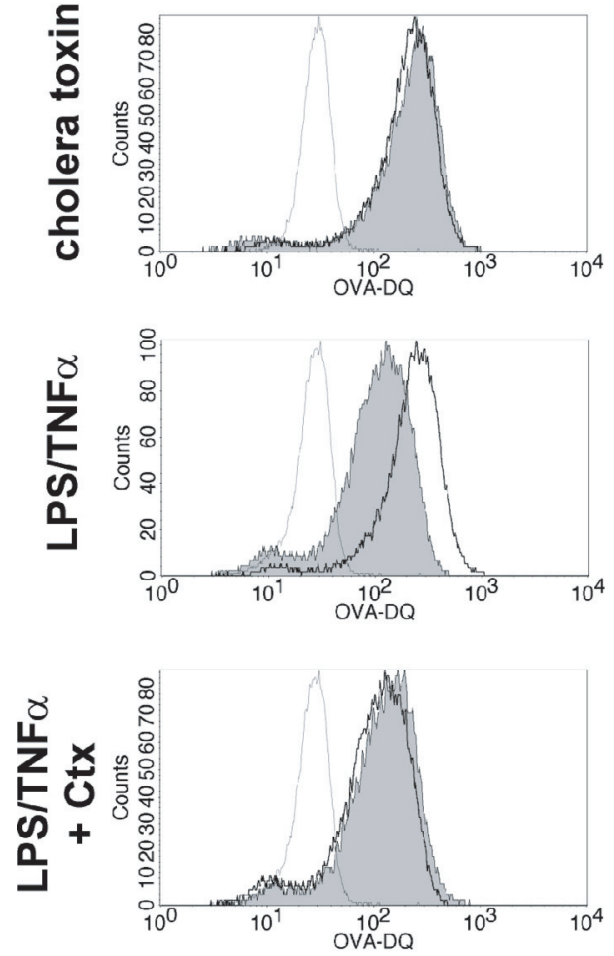

Figure 2. Influence of Ctx on endocytic capacity of MoDC. Uptake of $\mathrm{DQ}^{\mathrm{TM}}$ ovalbumin (OvaDQ) by MoDC after 30 min incubation at $39{ }^{\circ} \mathrm{C}$ was analysed by flow cytometry. DC maturation induced by LPS+TNF $\alpha$ caused a reduction in OvaDQ uptake. Dotted grey outline: control cell incubated at $4{ }^{\circ} \mathrm{C}$; black outline: upper two histograms - untreated control MoDC; bottom histogram - MoDC+LPS+TNF $\alpha$; solid grey histogram: MoDC + stimulants as indicated on the left. One representative experiment out of three is shown.

recognise phosphorylation of the NF- $\kappa \mathrm{B}$ inhibitor I $\mathrm{B} \alpha$ in Ctx-treated porcine DC. Additionally, we observed no I $\kappa \mathrm{B} \alpha$ degradation in response to Ctx. Further, we observed no phosphorylation of JNK, a kinase that regulates the activity of c-Jun within the transcription factor complex AP-1, in Ctx-treated cells (data not shown). 


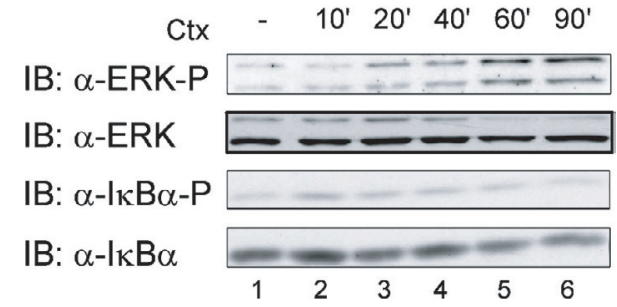

Figure 3. ERK activation in Ctx-treated DC. Monocyte-derived dendritic cells were either left untreated or stimulated with $10 \mu \mathrm{g} / \mathrm{mL}$ Ctx for 10-90 min. The protein extracts were subjected to SDS-PAGE and analysed in an immunoblot with antibodies against phosphoERK, ERK (loading control), phospho-IкB $\alpha$ and $I \kappa B \alpha$. The I $\mathrm{K} B \alpha$ protein was not degraded in response to Ctx. Data are representative of two independent experiments.

Therefore, we conclude that Ctx induces the signalling cascades leading to ERK activation, but has no effect on the regulation of the immediate early response transcription factors NF- $\kappa \mathrm{B}$ and AP-1.

\subsection{Ctx-primed DC inhibit T-cell proliferation}

To further analyse the functional effects of Ctx on MoDC, the capacity of Ctx-primed DC to stimulate T-cells was studied in a staphylococcal enterotoxin B (SEB) proliferation assay. As shown in Figure 4A, pre-treatment of MoDC with Ctx for $24 \mathrm{~h}$ significantly inhibited their T-cell stimulatory capacity. This effect was more prominent if DC were matured by adding LPS+TNF $\alpha$ and could be averted by adding GM1 to the cultures.

Antigen-specific proliferation assays with CSFV-antigens and lymphocytes of CSFV-primed donors revealed a similar suppression of stimulatory capacity of MoDC by Ctx. This effect was observed when co-cultures were infected with CSFV-virus (Fig. 4B) or mock, and also when the E2 (Fig. 4C) or the CSFVnon-structural protein 3 (NS3) proteins of CSFV (Fig. 4D) were used as antigens.

\subsection{Ctx does not induce apoptosis of DC or lymphocytes}

To analyse whether the reduced $\mathrm{T}$ cell proliferation induced by Ctx-primed MoDC was due to increased cell death, we performed Annexin-V FITC assays to detect apoptosis of MoDC and co-cultured lymphocytes. Figure $5 \mathrm{~A}$ demonstrates that neither Ctx or LPS+TNF $\alpha$ alone nor in combination caused an increased rate of MoDC apoptosis after 2, 6 or $24 \mathrm{~h}$. In contrast, $>80 \%$ of apoptotic cells were detected after $24 \mathrm{~h}$ of incubation with staurosporin.

Although Ctx did not directly cause apoptosis of MoDC, we hypothesised that Ctx-primed DC might mediate increased $\mathrm{T}$ cell death. Therefore, the percentage of apoptotic lymphocytes was measured in DC-lymphocyte co-cultures. As shown in Figure 5B, $24 \mathrm{~h}$ priming of MoDC with up to $10 \mu \mathrm{g} / \mathrm{mL}$ of Ctx did not result in a functional state of the DC that induced lymphocyte apoptosis. The presence of maturation factors did not alter the outcome of this experiment. Similarly, PI staining signifying necrosis and later stages of apoptosis was not influenced by Ctx (data not shown). Thus, apoptosis is unlikely to be the cause for the reduced proliferation rates.

\subsection{Ctx induces IL-10 and inhibits TNF $\alpha$ expression by porcine MoDC}

Since the reduced lymphocyte proliferation caused by Ctx-treatment of MoDC was not due to increased lymphocyte apoptosis, we examined whether pro- or antiinflammatory cytokines might play a role. The amounts of IL-10, IL-6 and IL-12 in culture supernatants of MoDC were studied by ELISA. As shown in Figure 6A, 
A

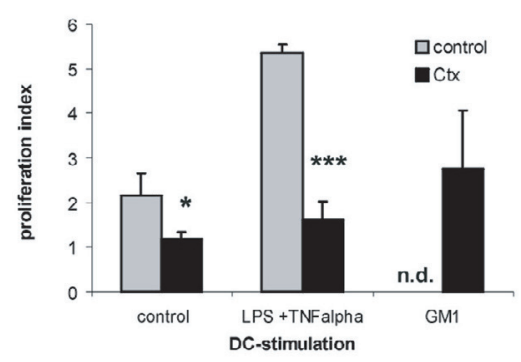

C

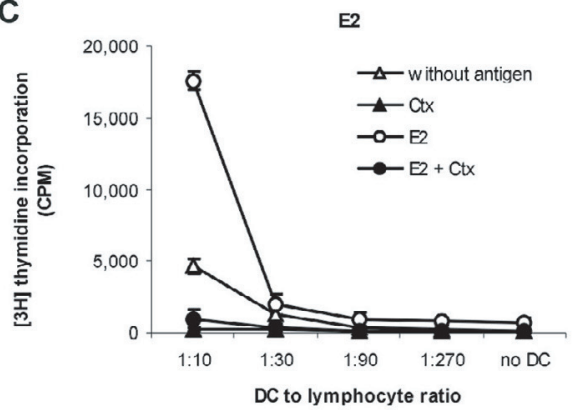

B
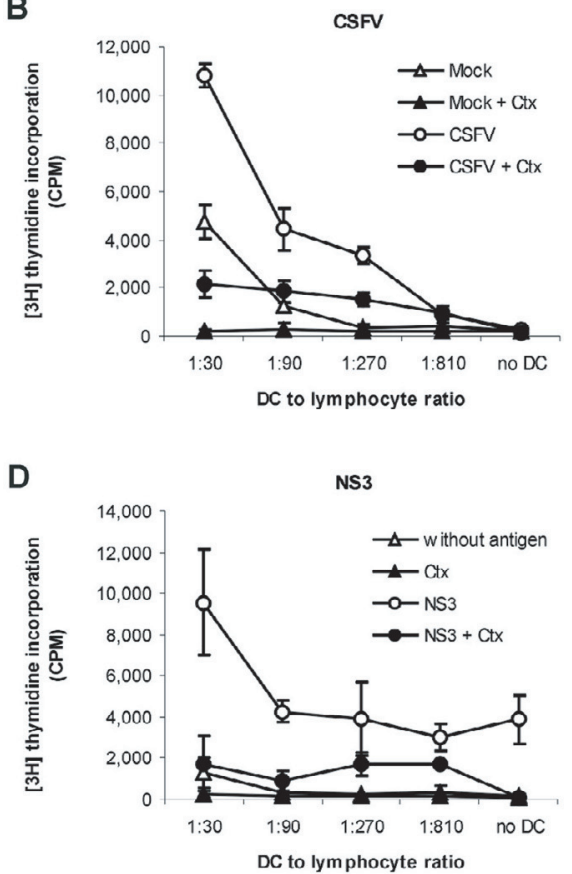

Figure 4. Inhibition of T-cell proliferation by Ctx-primed MoDC. (A) SEB-dependent proliferation assay. Porcine MoDC were left untreated or stimulated for $24 \mathrm{~h}$ with LPS+TNF $\alpha$ or GM1 in the presence or absence of Ctx. These cells were then loaded with SEB. The proliferative response of CD172a-depleted PBMC to these cells at a 1:10 ratio was determined after 4 days by BrdU-ELISA and is shown as the proliferation index. Mean \pm SD of triplicate wells from one representative out of five experiments are shown. n.d. $=$ not done, $* P<0.05$, $* * * P<0.001$. (B - D) Antigen-specific proliferation assays. After a total incubation time of $26 \mathrm{~h}$, antigen-pulsed MoDC with or without Ctx treatment were co-cultured with autologous lymphocytes in the presence of (B) CSFV (0.01 TCID $_{50}$ ) or mock antigen; (C) E2-antigen $(4 \mu \mathrm{g} / \mathrm{mL})$, or (D) NS3-antigen $(2 \mu \mathrm{g} / \mathrm{mL})$. Proliferative responses were determined after 4 days by $\left[\mathrm{H}^{3}\right]$ thymidine incorporation and are shown as counts per minute (CPM).

Ctx alone or in combination with either LPS, TNF $\alpha$, or IFN $\alpha+\mathrm{TNF} \alpha$ induced the secretion of IL-10 after $24 \mathrm{~h}$. IL-10 levels were the highest in cultures where Ctx was used in combination with LPS. LPS alone consistently induced low but detectable amounts of IL-10, whereas no IL-10 was found in cultures treated with $\mathrm{TNF} \alpha$, IFN $\alpha+\mathrm{TNF} \alpha$, or in control cultures. In contrast to IL-10, neither IL-6 nor IL-12 secretion could be induced by addition of maturation factors to MoDC cultures at any of the time points analysed (6 h, $24 \mathrm{~h}$, and $48 \mathrm{~h}$ ), even after stimulation with LPS (data not shown). This was not significantly altered by addition of Ctx.

As shown in Figure 6B, Ctx had a significant effect on TNF $\alpha$ secretion. Maturation factors LPS $+\mathrm{TNF} \alpha$ induced a release of TNF $\alpha$ after $6 \mathrm{~h}$. If Ctx was present in the MoDC cultures in addition to the maturation factors, $\mathrm{TNF} \alpha$ secretion was significantly reduced $(P<0.001)$. 

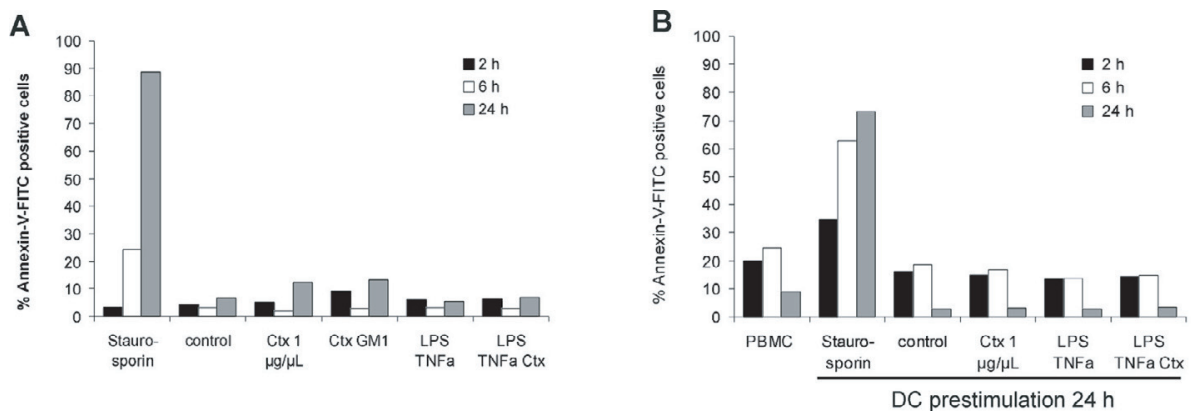

Figure 5. Ctx treatment of MoDC does not cause apoptosis of DC or of co-cultured lymphocytes. (A) Three-day MoDC cultures were stimulated with Ctx $(1 \mu \mathrm{g} / \mathrm{mL}), \mathrm{Ctx}+\mathrm{GM} 1(10 \mu \mathrm{g} / \mathrm{mL}), \mathrm{LPS}$ $(1 \mu \mathrm{g} / \mathrm{mL})+\mathrm{TNF} \alpha(10 \mathrm{ng} / \mathrm{mL})$, or Ctx + LPS + TNF $\alpha$ for 2,6 or $24 \mathrm{~h}$. (B) Three-day MoDC cultures were stimulated with Ctx $(1 \mu \mathrm{g} / \mathrm{mL}$ or $10 \mu \mathrm{g} / \mathrm{mL})$ and/or maturation factors for $24 \mathrm{~h}$ $(1 \mu \mathrm{g} / \mathrm{mL} \mathrm{Ctx} \pm 1 \mu \mathrm{g} / \mathrm{mL}$ LPS $+10 \mathrm{ng} / \mathrm{mL}$ TNF $\alpha)$. Cells were then harvested, treated with SEB and co-cultured with lymphocytes according to the protocol used for antigen-unspecific proliferation assays. Apoptosis of (A) MoDC and (B) lymphocytes was analysed by Annexin-V FITC-binding. Graphs show the percentage of apoptotic cells determined by FACS after the indicated periods of time. As a positive control, apoptosis was induced by addition of staurosporin $(1 \mu \mathrm{M})$.
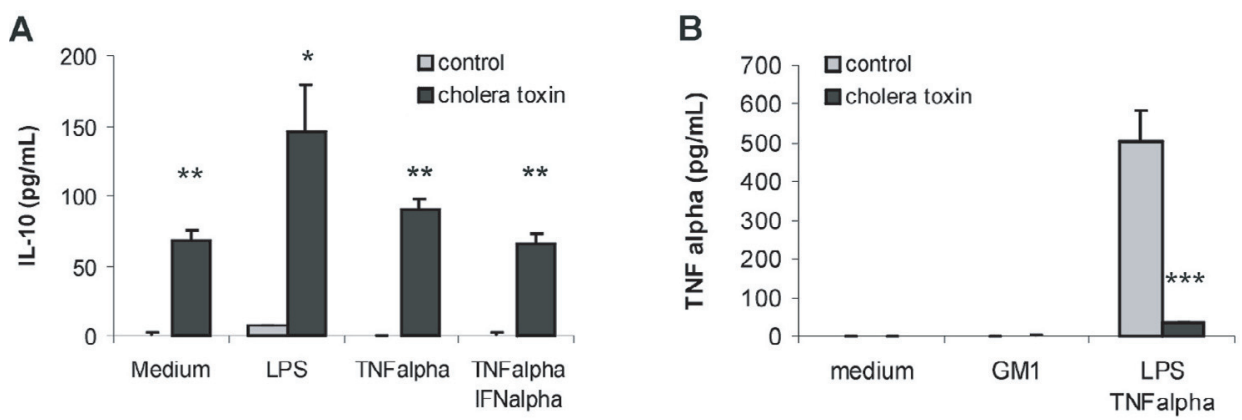

Figure 6. Cytokine production by Ctx-primed MoDC. (A) IL-10 levels in culture supernatants of MoDC stimulated with maturation factors (LPS, TNF $\alpha$, or IFN $\alpha+\mathrm{TNF} \alpha$ ) in the presence or absence of Ctx were determined by ELISA. Duplicate supernatants of three independent cultures were analysed after $24 \mathrm{~h}$. (B) TNF $\alpha$ production by MoDC cultures stimulated with Ctx, Ctx + GM1, LPS+TNF $\alpha$, or Ctx+LPS+TNF $\alpha$. Medium was exchanged twice after the first hour of incubation with the stimulants, and culture supernatants sampled after a total of $6 \mathrm{~h}$ were analysed by bioassay using PK15-15 cells. The results from one out of four independent experiments are shown. Differences between Ctx-treated and control cultures were tested for significance. ${ }^{*} P<0.05$, ** $P<0.01, * * * P<0.001$. 

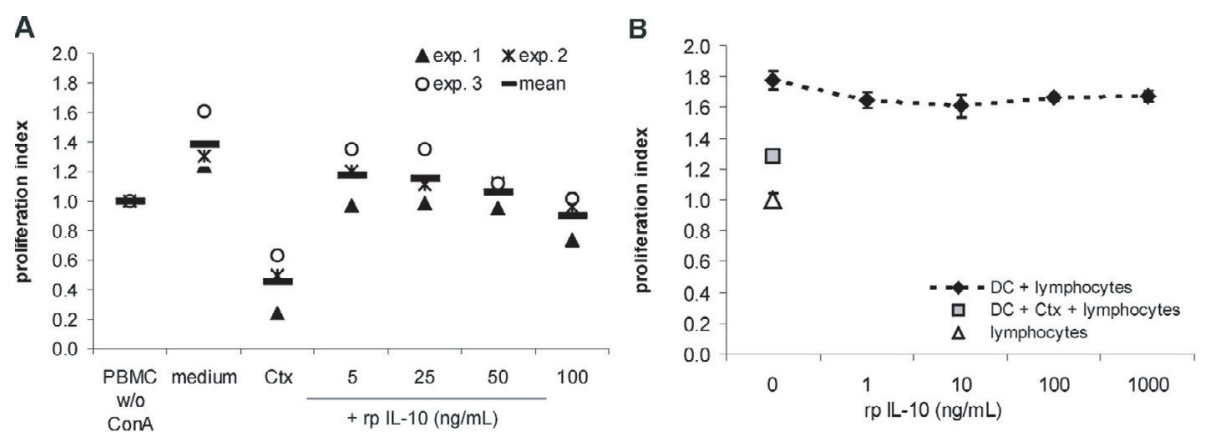

Figure 7. Influence of IL-10 in Ctx-induced inhibition of lymphocyte proliferation. (A) IL-10 induced inhibition of mitogen-driven lymphocyte proliferation. PBMC were cultured for 3 days in the presence of ConA and various concentrations of rp IL-10. Ctx $(1 \mu \mathrm{g} / \mathrm{mL})$ was used as positive control to suppress proliferation. Proliferation was determined by MTT-assay. Mean (bar) and data from individual experiments (exp., other symbols) of three independent experiments are shown. (B) IL-10 has no influence on stimulatory capacity of porcine MoDC. Porcine MoDC were stimulated for $24 \mathrm{~h}$ with Ctx or different concentrations of rp IL-10 and then loaded with SEB. The proliferative response of CD172a-depleted PBMC to the DC at a 1:10 ratio was determined after 3 days using an MTT-assay. One representative of three independent experiments is shown. The proliferation index was calculated by dividing absorption measured for the individual samples by absorption of unstimulated PBMC cultures.

\subsection{Altered cytokine secretion profiles contribute to the inhibitory effects of Ctx-primed MoDC on lymphocyte proliferation}

Since we observed increased IL-10 secretion by MoDC after Ctx stimulation, and IL-10 has been associated with a tolerogenic function of DC, we hypothesised that IL-10 might mediate the decreased $\mathrm{T}$ cell proliferation induced by Ctx-priming of MoDC. Therefore, we analysed whether IL-10 can directly inhibit proliferation of porcine lymphocytes. As shown in Figure 7A, the addition of IL-10 to mitogen-stimulated PBMC cultures caused a reduction of proliferation in a concentration-dependent manner. However, this effect was relatively weak, despite the high concentrations of IL-10 used in the assays. In addition, culture supernatants taken 6 or $24 \mathrm{~h}$ after MoDC stimulation with $\mathrm{Ctx}$ had no significant effect on PBMC proliferation (data not shown).
DC were stimulated with different IL10 concentrations before performing SEBproliferation assays in order to investigate possible autocrine effects of secreted IL10 on the MoDC. As shown in Figure 7B, IL-10 concentrations between $1 \mathrm{ng} / \mathrm{mL}$ and $1 \mu \mathrm{g} / \mathrm{mL}$ did not alter the stimulatory capacity of the DC.

We hypothesised that the decreased $\mathrm{TNF} \alpha$ secretion by Ctx-primed MoDC contributed to the reduced lymphocyte proliferation. Therefore rp TNF $\alpha$ was added to co-cultures of Ctx-primed DC and lymphocytes during the SEB-proliferation assays. Figure 8 demonstrates that the presence of $\mathrm{TNF} \alpha$ in the co-cultures could fully restore proliferation in a concentration dependent manner.

\subsection{Ctx-primed MoDC cannot inhibit lymphocyte proliferation that is induced by other MoDC}

To investigate whether Ctx-primed MoDC have direct suppressor effects on 


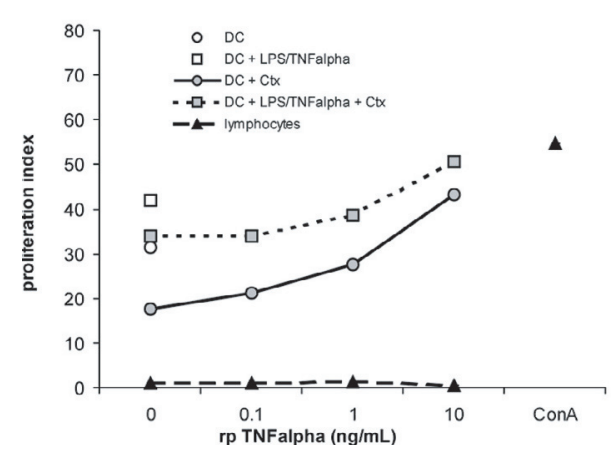

Figure 8. TNF $\alpha$ can restore lymphocyte proliferation in co-cultures with Ctx-primed MoDC. MoDC were treated with $\mathrm{rp} \mathrm{TNF} \alpha(10 \mathrm{ng} / \mathrm{mL})$ and LPS $(1 \mu \mathrm{g} / \mathrm{mL})$ to induce maturation or left untreated. Simultaneously, Ctx was added to some cultures as indicated. After $24 \mathrm{~h}, \mathrm{DC}$ were loaded with SEB and then co-cultured with lymphocytes at a 1:10 ratio in the presence of different concentrations of rpTNF $\alpha$. Proliferation was determined by BrdU-ELISA after 4 days and is given as a proliferation index. As controls, lymphocytes alone were stimulated with TNF $\alpha$ or ConA $(5 \mu \mathrm{g} / \mathrm{mL})$. Mean from one of two similar experiments is shown.

the function of other DC or lymphocytes and thus act as regulatory cells, lymphocyte proliferation in response to untreated MoDC in the presence of Ctx-treated MoDC was analysed. Stimulatory activity of untreated MoDC was not altered by the presence of Ctx-primed DC in the cultures, nor was the responsiveness of the $\mathrm{T}$ cells negatively modulated (Fig. 9). Thus, we could not obtain any evidence for an active suppressor function of Ctx-primed porcine MoDC.

\section{DISCUSSION}

In this study, the effects of cholera toxin (Ctx) on porcine monocyte-derived dendritic cells (MoDC) were analysed to explore the potential contribution of DC to the mucosal adjuvanticity of $\mathrm{Ctx}$ in the pig. Our main finding was a semi-mature,

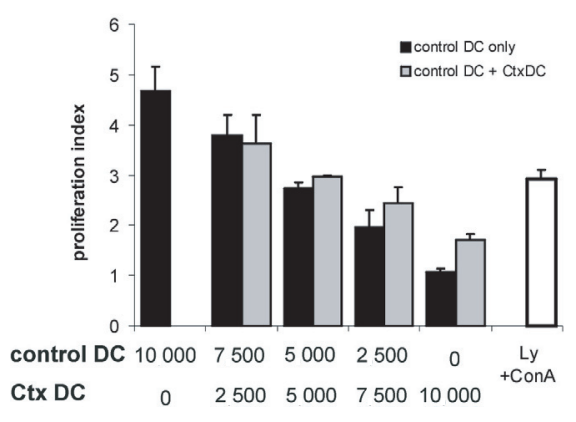

Figure 9. Ctx-primed MoDC do not suppress stimulatory capacity of untreated DC. Porcine MoDC were stimulated for $24 \mathrm{~h}$ with LPS+TNF $\alpha+\mathrm{Ctx}$ (Ctx DC) or LPS+TNF $\alpha$ only (control DC). MoDC were then loaded with $\mathrm{SEB}$, and the indicated numbers of control DC alone, or both control DC and Ctx DC were cocultured with 100000 lymphocytes. Lymphocytes (Ly) stimulated with ConA $(10 \mu \mathrm{g} / \mathrm{mL})$ served as positive control. Proliferation was determined after four days using a BrdUproliferation assay. One representative experiment out of six is shown.

non-stimulatory phenotype of MoDC after in vitro $\mathrm{Ctx}$-stimulation.

The phenotypic maturation of DC is typically associated with upregulation of molecules involved in the formation of the immunological synapse (e.g. MHCI/II, CD40, CD80, CD86) [5]. After Ctxstimulation, porcine MoDC acquired some features of mature DC, but retained others of immature DC, consistent with a "semimature" phenotype [31, 42]. Thus, expression of CD80/86 was increased, whereas expression of MHC-II and CD40 was decreased. Also, Ctx treatment caused a reduced expression of CCR1, a receptor for inflammatory chemokines which is typically down-regulated upon maturation to enable DC exit from inflamed tissue [44]. The endocytic capacity of DC, which decreases during the maturation process [43], was not modulated by Ctx stimulation. 
It has previously been shown that Ctx confers stimulatory effects through DC in some experimental settings $[18,19,26]$, and tolerogenic effects in others [28, 29]. This is possibly related to the activation and maturation stage of the DC at the time they encounter the Ctx. In our hands, Ctx induced the development of DC that were neither stimulatory nor actively suppressive. Ctx-primed porcine MoDC could neither induce $\mathrm{T}$ cell proliferation nor inhibit the proliferation of T cells activated by other DC present in the culture.

The observed phenotype of the DC in response to Ctx may largely be linked to an increase in intracellular cAMP. Ctx causes increased plasma levels of cAMP through the ADP-ribosyltransferase activity of the A-subunit after binding of the B-subunit to the cell surface via the ubiquitous GM1 ganglioside $[2,9,10,46]$. Consistent with our observations for Ctx, cAMP-elevating agents can directly increase IL-10 production, inhibit TNF $\alpha$ secretion and MHC-II and CD40 expression, and reduce stimulatory capacity in murine DC [25]. Although Ctx-primed porcine MoDC secreted higher amounts of IL-10, IL-10 alone had only weak effects on the proliferation of porcine T cells. Also, direct application of IL-10 to DC did not induce a non-stimulatory functional state. It is thus unlikely that the observed effects were primarily due to IL-10.

In porcine MoDC cultures, TNF $\alpha$ cooperates with IFN $\alpha$ or T cells to induce DC maturation [21]. Recently, it was shown that blocking $\mathrm{TNF} \alpha$ in human $\mathrm{DC}$ results in the generation of semi-mature DC [47]. Indeed, the stimulatory capacity of Ctx-primed MoDC seems to be largely due to a lack of TNF $\alpha$, since lymphocyte proliferation could be fully restored by adding rp $\mathrm{TNF} \alpha$ to the co-cultures in SEB-proliferation assays. This would also explain why Ctx-primed DC were not actively suppressive.
Activation of MAP kinases and other signal transduction pathways are important upstream events for the induction of DC maturation, migration or cytokine secretion [1,30]. Analysis of key intracellular signalling molecules in Ctx-stimulated MoDC revealed ERK1/2-activation, but no regulation of JNK or NFKB activity. The observed ERK1/2-phosphorylation might relate to the increase in intracellular cAMP in response to $\mathrm{Ctx}$. In other cells, e.g. PC12 cells, melanocytes, gastric and intestinal epithelial cells, elevation of cAMP results in ERK1/2-activation via a B-RAF - MEK1/2 pathway [12, 40, 51], which may also be responsible for $\mathrm{Ctx}$-induced ERK activation in porcine MoDC. Functionally, ERK1/2 activation has been associated with high levels of IL-10 secretion by DC [52], which is consistent with our findings. It has been previously demonstrated that functional DC maturation involves NFKB activation [39]. Kawamura et al. observed nuclear translocation of $\mathrm{NF} \kappa \mathrm{B}$ together with upregulation of maturation markers in Ctx-stimulated murine BM-DC [26]. The absence of NFKB activation in Ctx-stimulated porcine MoDC corroborates our observations of incomplete maturation and absence of T cell stimulatory function.

Relating to the question of Ctx in pig vaccination, our observations could explain the failure of Ctx to work as an oral adjuvant in pigs as described by Foss and Murtaugh [16]. However, if the nonstimulatory phenotype of Ctx-primed DC was largely caused by lack of $\mathrm{TNF} \alpha$, it seems possible that other cells such as macrophages or epithelial cells would supply pro-inflammatory cytokines in an in vivo situation [10]. Notably, the F4fimbriae co-administered with Ctx by Verdonck et al. [48] also directly act as mucosal antigens with the potential capacity to modulate DC phenotype. Further studies on intestinal DC or on co-cultures of DC 
and intestinal epithelial cells are necessary to analyse this aspect.

\section{ACKNOWLEDGEMENTS}

We thank S. Schneider, C. Balmelli, L. Guzylack-Piriou, V. Tâche, and H. Gerber for helpful discussions and technical assistance.

D. Bimczok was financially supported by the Deutsche Forschungsgemeinschaft, GRK 745 "Mucosal Host-Pathogen Interactions", the European Union, 5th framework programme "Healthypigut" QLK5-CT-2000-00522, and 6th framework programme "Feed for Pig Health" FOOD-CT 2004-506144. H. Rau was supported by the Swiss Federal Office for Education and Science (Project BBW-No. 00.0635 linked to EU project QLK2-CT-2001-01374).

\section{REFERENCES}

[1] Ardeshna K.M., Pizzey A.R., Devereux S., Khwaja A., The PI3 kinase, p38 SAP kinase, and NF-kappaB signal transduction pathways are involved in the survival and maturation of lipopolysaccharide-stimulated human monocyte-derived dendritic cells, Blood (2000) 96:1039-1046.

[2] Bagley K.C., Abdelwahab S.F., Tuskan R.G., Fouts T.R., Lewis G.K., Cholera toxin and heat-labile enterotoxin activate human monocyte-derived dendritic cells and dominantly inhibit cytokine production through a cyclic AMP-dependent pathway, Infect. Immun. (2002) 70:5533-5539.

[3] Balmelli C., Ruggli N., McCullough K., Summerfield A., Fibrocytes are potent stimulators of anti-virus cytotoxic T cells, J. Leukoc. Biol. (2005) 77:923-933.

[4] Banchereau J., Steinman R.M., Dendritic cells and the control of immunity, Nature (1998) 392:245-251.

[5] Banchereau J., Briere F., Caux C., Davoust J., Lebecque S., Liu Y.J., Pulendran B., Palucka K., Immunobiology of dendritic cells, Annu. Rev. Immunol. (2000) 18:767811.

[6] Bertoni G., Kuhnert P., Peterhans E., Pauli U., Improved bioassay for the detection of porcine tumor necrosis factor using a homologous cell line: PK(15), J. Immunol. Methods (1993) 160:267-271.
[7] Bilsborough J., Viney J.L., Gastrointestinal dendritic cells play a role in immunity, tolerance, and disease, Gastroenterology (2004) 127:300-309.

[8] Carrasco C.P., Rigden R.C., Schaffner R., Gerber H., Neuhaus V., Inumaru S., Takamatsu H., Bertoni G., McCullough K.C., Summerfield A., Porcine dendritic cells generated in vitro: morphological, phenotypic and functional properties, Immunology (2001) 104:175-184.

[9] Cassel D., Selinger Z., Mechanism of adenylate cyclase activation by cholera toxin: inhibition of GTP hydrolysis at the regulatory site, Proc. Natl. Acad. Sci. USA (1977) 74:3307-3311.

[10] Cox E., Verdonck F., Vanrompay D., Goddeeris B., Adjuvants modulating mucosal immune responses or directing systemic responses towards the mucosa, Vet. Res. (2006) 37:511-539.

[11] Denizot F., Lang R., Rapid colorimetric assay for cell growth and survival. Modifications to the tetrazolium dye procedure giving improved sensitivity and reliability, J. Immunol. Methods (1986) 89:271277.

[12] Dumaz N., Marais R., Integrating signals between cAMP and the RAS/RAF/MEK/ERK signalling pathways. Based on the anniversary prize of the Gesellschaft fur Biochemie und Molekularbiologie Lecture delivered on 5 July 2003 at the Special FEBS Meeting in Brussels, FEBS J. (2005) 272:3491-3504.

[13] Elson C.O., Dertzbaugh M.T., Mucosal adjuvants, in: Ogra P.L., Mestecky J., Lamm M.E., Strober A., McGhee J.R., Bienenstock J. (Eds.), Handbook of Mucosal Immunology, San Diego, Academic Publishers, 1994, pp. 391-401.

[14] Eriksson K., Holmgren J., Recent advances in mucosal vaccines and adjuvants, Curr. Opin. Immunol. (2002) 14: 666-672.

[15] Fleeton M., Contractor N., Leon F., He J., Wetzel D., Dermody T., Iwasaki A., Kelsall B., Involvement of dendritic cell subsets in the induction of oral tolerance and immunity, Ann. NY Acad. Sci. (2004) 1029:60-65.

[16] Foss D.L., Murtaugh M.P., Mucosal immunogenicity and adjuvanticity of cholera toxin in swine, Vaccine (1999) 17:788-801.

[17] Foss D.L., Zilliox M.J., Murtaugh M.P., Differential regulation of macrophage interleukin-1 (IL-1), IL-12, and CD80-CD86 by two bacterial toxins, Infect. Immun. (1999) 67:5275-5281. 
[18] Gagliardi M.C., Sallusto F., Marinaro M., Langenkamp A., Lanzavecchia A., De Magistris M.T., Cholera toxin induces maturation of human dendritic cells and licences them for Th2 priming, Eur. J. Immunol. (2000) 30:2394-2403.

[19] Gagliardi M.C., De Magistris M.T., Maturation of human dendritic cells induced by the adjuvant cholera toxin: role of cAMP on chemokine receptor expression, Vaccine (2003) 21:856-861.

[20] Guzylack-Piriou L., Balmelli C., McCullough K.C., Summerfield A., Type-A $\mathrm{CpG}$ oligonucleotides activate exclusively porcine natural interferon-producing cells to secrete interferon-alpha, tumour necrosis factor-alpha and interleukin-12, Immunology (2004) 112:28-37.

[21] Guzylack-Piriou L., Piersma S., McCullough K., Summerfield A., Role of natural interferon-producing cells and $\mathrm{T}$ lymphocytes in porcine monocyte-derived dendritic cell maturation, Immunology (2006) 118:78-87.

[22] Jang M.H., Kweon M.N., Hiroi T., Yamamoto M., Takahashi I., Kiyono H., Induction of cytotoxic $\mathrm{T}$ lymphocyte responses by cholera toxin-treated bone marrow-derived dendritic cells, Vaccine (2003) 21:1613-1619.

[23] Johansson E., Domeika K., Berg M., Alm G.V., Fossum C., Characterisation of porcine monocyte-derived dendritic cells according to their cytokine profile, Vet. Immunol. Immunopathol. (2003) 91:183-197.

[24] Kaden V., Lange E., Fischer U., Strebelow G., Oral immunisation of wild boar against classical swine fever: evaluation of the first field study in Germany, Vet. Microbiol. (2000) 73:239-252.

[25] Kambayashi T., Wallin R.P., Ljunggren H.G., cAMP-elevating agents suppress dendritic cell function, J. Leukoc. Biol. (2001) 70:903-910.

[26] Kawamura Y.I., Kawashima R., Shirai Y., Kato R., Hamabata T., Yamamoto M., Furukawa K., Fujihashi K., McGhee J.R., Hayashi H., Dohi T., Cholera toxin activates dendritic cells through dependence on GM1-ganglioside which is mediated by NF-kappaB translocation, Eur. J. Immunol. (2003) 33:3205-3212.

[27] Knoetig S.M., Summerfield A., Spagnuolo-Weaver M., McCullough K.C., Immunopathogenesis of classical swine fever: role of monocytic cells, Immunology (1999) 97:359-366.
[28] Lavelle E.C., McNeela E., Armstrong M.E., Leavy O., Higgins S.C., Mills K.H., Cholera toxin promotes the induction of regulatory $\mathrm{T}$ cells specific for bystander antigens by modulating dendritic cell activation, J. Immunol. (2003) 171:2384-2392.

[29] Lavelle E.C., Jarnicki A., McNeela E., Armstrong M.E., Higgins S.C., Leavy O., Mills K.H., Effects of cholera toxin on innate and adaptive immunity and its application as an immunomodulatory agent, J. Leukoc. Biol. (2004) 75:756-763.

[30] Luft T., Maraskovsky E., Schnurr M., Knebel K., Kirsch M., Gorner M., Skoda R., Ho A.D., Nawroth P., Bierhaus A., Tuning the volume of the immune response: strength and persistence of stimulation determine migration and cytokine secretion of dendritic cells, Blood (2004) 104:1066-1074.

[31] Lutz M.B., Schuler G., Immature, semimature and fully mature dendritic cells: which signals induce tolerance or immunity? Trends Immunol. (2002) 23:445-449.

[32] Lycke N., From toxin to adjuvant: the rational design of a vaccine adjuvant vector, CTA1-DD/ISCOM, Cell. Microbiol. (2004) $6: 23-32$.

[33] Mitragotri S., Immunization without needles, Nat. Rev. Immunol. (2005) 5:905-916.

[34] Naumann M., Scheidereit C., Activation of NF-kappa B in vivo is regulated by multiple phosphorylations, EMBO J. (1994) 13:4597-4607.

[35] Paillot R., Laval F., Audonnet J.C., Andreoni C., Juillard V., Functional and phenotypic characterization of distinct porcine dendritic cells derived from peripheral blood monocytes, Immunology (2001) 102:396-404.

[36] Rau H., Revets H., Balmelli C., McCullough K.C., Summerfield A., Immunological properties of recombinant classical swine fever virus NS3 protein in vitro and in vivo, Vet. Res. (2006) 37:155-168.

[37] Raymond C.R., Wilkie B.N., Th-1/Th-2 type cytokine profiles of pig T-cells cultured with antigen-treated monocyte-derived dendritic cells, Vaccine (2004) 22:1016-1023.

[38] Raymond C.R., Sidahmed A.M., Wilkie B.N., Effects of antigen and recombinant porcine cytokines on pig dendritic cell cytokine expression in vitro, Vet. Immunol. Immunopathol. (2006) 111:175-185.

[39] Rescigno M., Martino M., Sutherland C.L., Gold M.R., Ricciardi-Castagnoli P., Dendritic cell survival and maturation are 
regulated by different signaling pathways, J. Exp. Med. (1998) 188:2175-2180.

[40] Rudolph J.A., Poccia J.L., Cohen M.B., Cyclic AMP activation of the extracellular signal-regulated kinases 1 and 2: implications for intestinal cell survival through the transient inhibition of apoptosis, J. Biol. Chem. (2004) 279:14828-14834.

[41] Ruggli N., Tratschin J.D., Mittelholzer C., Hofmann M.A., Nucleotide sequence of classical swine fever virus strain Alfort/187 and transcription of infectious RNA from stably cloned full-length cDNA, J. Virol. (1996) 70:3478-3487.

[42] Rutella S., Danese S., Leone G., Tolerogenic dendritic cells: Cytokine modulation comes of age, Blood (2006) 108:1435-1440.

[43] Sallusto F., Cella M., Danieli C., Lanzavecchia A., Dendritic cells use macropinocytosis and the mannose receptor to concentrate macromolecules in the major histocompatibility complex class II compartment: downregulation by cytokines and bacterial products, J. Exp. Med. (1995) 182:389-400.

[44] Sallusto F., Schaerli P., Loetscher P., Schaniel C., Lenig D., Mackay C.R., Qin S., Lanzavecchia A., Rapid and coordinated switch in chemokine receptor expression during dendritic cell maturation, Eur. J. Immunol. (1998) 28:2760-2769.

[45] Strobel S., Mowat A.M., Immune responses to dietary antigens: oral tolerance, Immunol. Today (1998) 19:173-181.

[46] Torgersen M.L., Skretting G., van Deurs B., Sandvig K., Internalization of cholera toxin by different endocytic mechanisms, J. Cell Sci. (2001) 114:3737-3747.
[47] Van Lieshout A.W., Barrera P., Smeets R.L., Pesman G.J., van Riel P.L., van den Berg W.B., Radstake T.R., Inhibition of TNF alpha during maturation of dendritic cells results in the development of semimature cells: a potential mechanism for the beneficial effects of TNF alpha blockade in rheumatoid arthritis, Ann. Rheum. Dis. (2005) 64:408-414.

[48] Verdonck F., Snoeck V., Goddeeris B.M., Cox E., Cholera toxin improves the F4(K88)-specific immune response following oral immunization of pigs with recombinant FaeG, Vet. Immunol. Immunopathol. (2005) 103:21-29.

[49] Vincent I.E., Carrasco C.P., Herrmann B., Meehan B.M., Allan G.M., Summerfield A., McCullough K.C., Dendritic cells harbor infectious porcine circovirus type 2 in the absence of apparent cell modulation or replication of the virus, J. Virol. (2003) 77:1328813300 .

[50] Von Niederhausern B., Bertoni G., Hertig C., Pfister H., Peterhans E., Pauli U., Cloning and expression in mammalian cells of porcine tumor necrosis factor alpha: examination of biological properties, Vet. Immunol. Immunopathol. (1993) 38:57-74.

[51] Wessler S., Rapp U.R., Wiedenmann B., Meyer T.F., Schoneberg T., Hocker M., Naumann M., B-Raf/Rap1 signaling, but not c-Raf-1/Ras, induces the histidine decarboxylase promoter in Helicobacter pylori infection, FASEB J. (2002) 16:417-419.

[52] Xia C.Q., Peng R., Beato F., ClareSalzler M.J., Dexamethasone induces IL-10producing monocyte-derived dendritic cells with durable immaturity, Scand. J. Immunol. (2005) 62:45-54. 